discernible negative difference from the patient's perspective in the clinical sessions. This may be due to the difficulties in therapeutic alliance using the telehealth platform. We appreciate that there are a number of confounding factors, especially the effect of COVID-19 isolation. Telehealth is a useful addition to our assessment and treatment paradigms and its use should continue; however, we should be aware of the potential negative effect on therapeutic alliance.

\section{What happens to people admitted to a specialist dementia unit in the west of Scotland?}

Andrew Donaldson*, Craig Patrick, Lindsay Short and Helen Maginnis

NHS Lanarkshire

${ }^{\star}$ Corresponding author.

doi: $10.1192 /$ bjo.2021.840

Aims. Recent local research examined mortality rates following admission to a dementia ward. We wanted to expand on this work and include other important health outcomes for patients admitted to our specialist in-patient dementia unit in the west of Scotland. This would provide a comprehensive overview of our in-patient population, aid service review and improve care. We hypothesised that patients admitted would be physically frail, have a significant mortality rate and would likely require long-term care post discharge.

Method. The clinical notes for each admission to the unit for one year were examined (total 62). We extracted data from a number of different areas such as demographics, mortality rates, discharge destination, readmission rates and prescribed medications.

Result. $60 \%$ had an Alzheimer's/mixed dementia diagnosis. Average length of stay was 64 days. $62 \%$ were discharged to a care home (50\% of this total had lived at home prior to admission), $18 \%$ to complex care and $20 \%$ to the community. $66 \%$ were prescribed an antipsychotic and the average number of medications was $8.4 .35 \%$ had a readmission under general medicine within a year of discharge. $19 \%$ died whilst an inpatient and a further $30 \%$ had died one year post-discharge (total one-year mortality of $44 \%$ ). Conclusion. People admitted to our dementia unit are physically frail, with only $20 \%$ returning to live in the community, $35 \%$ being readmitted to a general medical ward within a year of discharge and $44 \%$ dying during the admission or within a year of discharge. We need to bear these results in mind when considering if hospital admission is appropriate and ultimately further develop our skills in palliative and end of life care in order to provide those people admitted to our dementia unit (and those who remain at home) with the highest standard of care.

\section{Audit of pharmacological management of borderline personality disorder as per NICE clinical guidelines CG78}

Bethany Dudley ${ }^{1 \star}$, Shakina Bellam ${ }^{1}$ and Andrew Lawrie ${ }^{2}$

${ }^{1}$ Monkwearmouth Hospital, Cumbria Northumberlnd Tyne, Wear

NHS Foundation Trust and ${ }^{2}$ Monkwearmouth Hospital, Sunderland, Cumbria, Northumberland Tyne Wear NHS Foundation Trust ${ }^{*}$ Corresponding author.

doi: 10.1192/bjo.2021.841

Aims. To audit the current practice of pharmacological management of Borderline Personality Disorder with NICE Clinical guideline [CG78]: Borderline personality disorder:
Objectives:

23 patient records were analysed in the last 18 months with a diagnosis of EUPD to compare current practice against NICE clinical guidance. (2009)

Standards:

When prescribing

1) Use a single drug.

2) Use the minimum effective dose.

3) Agree with the person the target symptoms, monitoring arrangements and anticipated duration of treatment. Antipsychotic drugs should not be used for medium, long term treatment.

Indication:

4) Drug treatment should not be used specifically for borderline personality disorder or for the individual symptoms or behaviour associated. (Repeated self-harm, marked emotional instability, risk taking behaviour and transient psychotic symptoms).

5) Short-term use of sedative medication may be considered cautiously as part of the overall treatment plan in a crisis. The duration of treatment should be no longer than 1 week.

6) When considering drug treatment, provide the person with written material about the drug. This should include evidence for the drug's effectiveness in the treatment of borderline personality disorder and for any comorbid condition, and potential harm.

Review:

7) Review the effectiveness and tolerability of previous and current treatments.

8) Discontinue ineffective treatments.

Background. Borderline Personality Disorder is common in psychiatric settings with a reported prevalence of $20 \%$.

As per NICE Guidance (CG 78), no medications have been found effective for the longer term treatment of personality difficulties.

This audit was carried out to review if patients were offered psychiatric reviews to discuss the medications they are using, the effectiveness of these, and any potential side effects.

Result. Good practice compliance of $90-100 \%$ was noted where $>90 \%$ compliance was seen in areas where the effectiveness and tolerability of current and previous medication was reviewed by the clinicians under Structured Clinical Management. Also was noted that antipsychotics were not used for medium to long term in patients with Borderline Personality Disorder in the cohort.

The following areas were non-compliant with the NICE recommendations where a compliance $<79 \%$ has been achieved.

When prescribing, use a single drug (avoid polypharmacy), agree target symptoms, monitoring and duration, provide written information, discuss evidence for effectiveness in treatment of borderline personality disorder.

Partial compliance was achieved (80-89\%) with use of sedatives for less than 1 week and discontinuation of ineffective treatment.

Conclusion. Distribute key cards to clinicians.

Provide written information to patients.

Re-audit in 6 months.

An audit on admission clerkings across Lancashire and South Cumbria NHS Foundation Trust (LSCFT)

Sophie Edgell ${ }^{1 \star}$, Ahmed Sultan ${ }^{1}$ and Mohammed Hussain ${ }^{2}$

${ }^{1}$ Chorley Mental Health Inpatient Unit and ${ }^{2}$ The Harbour

${ }^{\star}$ Corresponding author.

doi: 10.1192/bjo.2021.842 
Aims. Following a Care Quality Commission (CQC) outcome showing that capacity assessments were not routinely completed on admission of patients we decided to complete an audit on current practice. We planned to review admission clerkings at Chorley Mental Health Inpatient Unit to assess quality, with the overall aim of putting measures in place to improve standards. We planned to make the results reflective of all psychiatry wards within Lancashire and South Cumbria NHS Foundation Trust (LSCFT) with the addition of a qualitative survey.

Background. We are aware the standard of clerkings can vary and affect patient care. CQC outcome showed that that capacity assessment was not routinely documented and consultants have stated that clinical impressions are rarely documented in junior doctor clerkings. This audit allowed us to objectively assess these observations. We believed the results may show common themes throughout psychiatric practice more generally.

Method. The gold standard was a 20 item list of expected components of a clerking, based on trust guidelines. A snapshot of current inpatients $(n=30)$ on $31 / 10 / 19$ was taken. An Excel sheet was used for information gathering. Data were analysed and graphs created. A qualitative questionnaire on current practice was sent to trainees $(n=8)$ on different sites for an overview of practice across LSCFT. Therefore, a mixed-methods model was employed.

Result. Items with the highest completion included clerking within 6 hours, face-to-face review with consultant completed within a week and current medication documentation. The items with the lowest completion included clinical impression documentation, bloods completed within 24 hours and documentation of capacity assessment and smoking/substances history. Common factors between clerkings with fewer completed items included poor patient engagement and patient transfer from another ward.

Qualitative survey $(n=8)$ showed that junior doctors across the health board are not using uniform methods for capacity documentation or an official checklist for clerking.

Conclusion. We concluded that the low rate of capacity assessment completion was an important finding due to legal implications, and that there should be a uniform place for documentation of this. Physical health consequences of other missing components were explored. We will introduce standardisation of capacity assessment documentation and use of a clerking checklist, before re-auditing. The results were presented at local teaching and recommendations sent to Site Tutors for inclusion in local inductions.

\section{A service evaluation and improvement project:} outcomes of older adult functional referrals to the North Derbyshire Liaison Team

Maha Ejaz ${ }^{1 \star}$, Joseph Atkins ${ }^{1}$, Bushra Azam ${ }^{2}$ and Stuart Laverack ${ }^{2}$

${ }^{1}$ University of Sheffield and ${ }^{2}$ Derbyshire Healthcare NHS Foundation Trust

${ }^{\star}$ Corresponding author.

doi: 10.1192/bjo.2021.843

Aims. This project aims to examine a group of service users over the age of 65 with functional presentations, who were assessed by the Liaison Psychiatry team between June 2018 and 2019.

Hypotheis: We believe that there is a need for a community crisis service for the older adult North Derbyshire population with functional presentations.

Background. Due to the lack of community crisis services for patients over 70, it was felt that a significant number of these patients were admitted to inpatient psychiatric units from medical wards who would benefit from crisis intervention instead. We wanted to see the clinical outcomes of this population, referred to the liaison team, determining whether this was significant concern. If this need is established, based on the data collected, this will enable the trust to look into starting a service for this age group to provide care in their own home. In turn, it will help to reduce unnecessary admissions to acute mental health wards and reduce stays in the general hospital - preventing consequences associated with long term hospital stays.

Method. Retrospective analysis using PARIS notes of 366 patients referred to the liaison team were scrutinised to determine the assessment diagnosis and outcome of patients with functional conditions. The inclusion criteria were patients over the age of 65 referred with functional psychiatric illnesses between June 2018 and 2019. We excluded 84 patients assessed to have delirium or organic presentations from our analysis. Data were collected and analysed using Excel.

Result. Among the referrals to the liaison team, the majority of patients were referred with mood disorders followed by self-harm, psychosis and anxiety. Although the majority of patients were referred back to either the community mental health team or primary care, $11 \%$ of the sample were admitted to inpatient psychiatric units. This number may have been lower and admission may have been avoided if a community crisis service was in place for this population.

Conclusion. In conclusion, the data support our initial concerns that there is a need for crisis services for this age group with functional presentations. There is ongoing discussions around a need to develop this service and therefore our results will contribute to the development of an older adult functional service in Derbyshire.

Listen to hard topics with soft ears - domestic violence and family carer; a survey of referrals to a MHIDD forensic mental health service in Ireland

Mohamed Elhassan Elamin*, Anthony Kearns and Aidan Cooney

National Forensic Mental Health Services

${ }^{*}$ Corresponding author.

doi: 10.1192/bjo.2021.844

Aims. A number of studies sought to explore and define families needs, experiences and concerns associated with being a carer for a detained person and their interaction with Forensic services (McKeown et al, 1995, MacInnes et al, 2002, Tsang et al, 2002, Absalom et al, 2012 Horberg et al, 2015).

Relatives can be victims of the service user's offence (Ferriter \& Huband, 2003, Tsang et al 2002), and may even blame the service user for their behaviour (Barrowclough et al., 2005). Service user becomes violent and aggressive family members are less likely to be motivated to participate, due to the service user's behaviour (MacInnes, 2000).

An initial domestic violence survey of in-patient case files found that in $66 \%$ of the patients files, there were reported incidents of domestic violence in family caring relationships prior to the index offence and subsequent admission to NFMHS (Cooney, 2018).

Method. A quantitative methodology was used. A domestic violence survey of referrals was conducted of 100 referrals to the National Forensic Mental Health Services - Mental Health and Intellectual \& Development Disability Services between 2016-2019.

Result. 22\% of the referrals reported Domestic Violence in the family care-giving relationships. 